\title{
The ImageJ Ecosystem: An Open and Extensible Platform for Biomedical Image Analysis.
}

\author{
Curtis T. Rueden ${ }^{1}$, and Kevin W. Eliceiri ${ }^{1,2}$ \\ 1. Laboratory for Optical and Computational Instrumentation, University of Wisconsin at Madison, \\ Madison, Wisconsin, USA. \\ 2. Morgridge Institute for Research, Madison, Wisconsin, USA.
}

Biological imaging has greatly advanced over the last thirty years with the now unprecedented ability to track biological phenomena in high resolution in physiologically relevant conditions over time and in space. As these imaging technologies mature and become main stream tools for the bench biologist there is great need for improved software tools that drive the informatics workflow of the imaging process from acquisition and image analysis to visualization and dissemination. To best meet the workflow challenges, these tools need to be freely available, open source, and transparent in their development and deployment. In particular it is clear that given the complexity, and heterogeneity of the modern image dataset, there cannot be a single software solution. Different imaging processing and visualization approaches need access not only to the data but also to each other. There needs to be compatibility not only in file import and export but interoperability in preserving and communicating what was done to the image. There is a great opportunity in achieving this interoperability, tools that can talk to each other not only enable new biological discovery but also efficiencies in sharing code and in many cases more precise workflows. We present our efforts towards interoperability and extensibility in the ImageJ consortium. We are actively developing key software libraries like ImgLib and ImageJ Ops that are utilized to analyze and visualize biological image data, to the developmental benefit of not only of the applications but the libraries themselves. We also overview the two major development efforts of the ImageJ [1,2] family of image analysis, FIJI [3] and ImageJ2 [4].

The ImageJ distribution Fiji ("Fiji Is Just ImageJ") was conceived to address the need of an easy-toinstall image-processing package for biologists, based on ImageJ. Always inspired by researchers' needs, Fiji bundles many ImageJ plugins and offers tutorials and documentation in the ever-growing Fiji Wiki. To facilitate the interaction between biologists and programming experts, Fiji has an update function that allows for a rapid turn-around time between development and usage of Fiji components.

Fiji not only caters for regular users but also for advanced ones, offering powerful scripting languages in addition to the ImageJ macro language. Expert programmers benefit from Fiji's Script Editor which allows to edit and run not only scripts but also Java plugins without the need to leave ImageJ. To avoid duplication of efforts and to provide better separation of concerns, Fiji collaborates closely with other projects such as Bio-Formats, ImageJ2 and ImgLib.

Any successful software project, after a period of sustained growth and the addition of functionality outside the scope of the program's original intent, will benefit from a subsequent period of scrutiny and refactoring, and Image $\mathrm{J}$ is no exception. Image $\mathrm{J} 2$ is a new version of Image $\mathrm{J}$ seeking to strengthen both the software and its community. Internally, it is a total redesign of ImageJ, but it is backwards compatible with ImageJ 1.x via a "legacy layer" and features a user interface closely modeled after the original. Under the hood, ImageJ 2 completely isolates the image processing logic from the graphical user interface (UI), allowing Image 2 plugins to be used in many contexts, including headless in the cloud or on a server such as OMERO, or from within another application such as KNIME, ICY or 
CellProfiler (a Python application). Image 2 has an N-dimensional data model driven by the powerful ImgLib2 library, which supports image data expressed in an extensible set of numeric and non-numeric types, and accessed from an extensible set of data sources. ImageJ 2 is driven by a state-of-the-art, collaborative development process, including version control, unit testing, automated builds via a continuous integration system, a bug tracker and more. We are collaborating closely with related projects including Fiji, CellProfiler, KNIME, Bio-Formats and OMERO, and are striving to deliver a coherent software stack reusable throughout the life sciences community and beyond. The result is welldesigned, community-driven software accessible to users yet powerful enough for programmers. ImageJ2 is available separately but the preferred distribution for biologists is via FIJI where it is prebundled.

Meeting current and future needs of scientific image analysis requires a flexible and extensible data model, supporting arbitrary dimensions, data types and image sizes. To this end, we chose to use the ImgLib2 library, which provides an extensible, interface-driven design that supports numeric and nonnumeric data types. It also provides great flexibility regarding the source and structure of data. Out of the box, ImgLib2 provides several data sources and organizations, including use of N-dimensional array "cells." However, the library remains general enough that alternative structures are also feasible.

Another very recent ImageJ2/FIJI development is on the image processing side with the development of the Ops framework (Figure 1). ImageJ Ops is a framework for reusable image processing operations that extends Java's mantra of "write once, run anywhere" to image processing algorithms. We describe how Ops helps with minimizing complexity, how it helps maintain good performance by allowing for extensible optimization, and allows for effective sharing and reuse of imaging analysis functions [5].

\section{References:}

[1] CA Schneider, WS Rasband, KW Eliceiri. Nature Methods 9 (2012), p. 671.

[2] ET Arena, et al, Wiley Interdiscip Rev Dev Biol. 6 (2017), p 260.

[3] J Schindelin, et al, Nature Methods 9 (2012), p 676.

[4] J Schindelin, et al, Mol Reprod Dev. 82 (2015), p 518.

[5] The authors acknowledge funding from NIH grants R03 EB008516 and RC2 GM092519 and from the Morgridge Institute for Research.

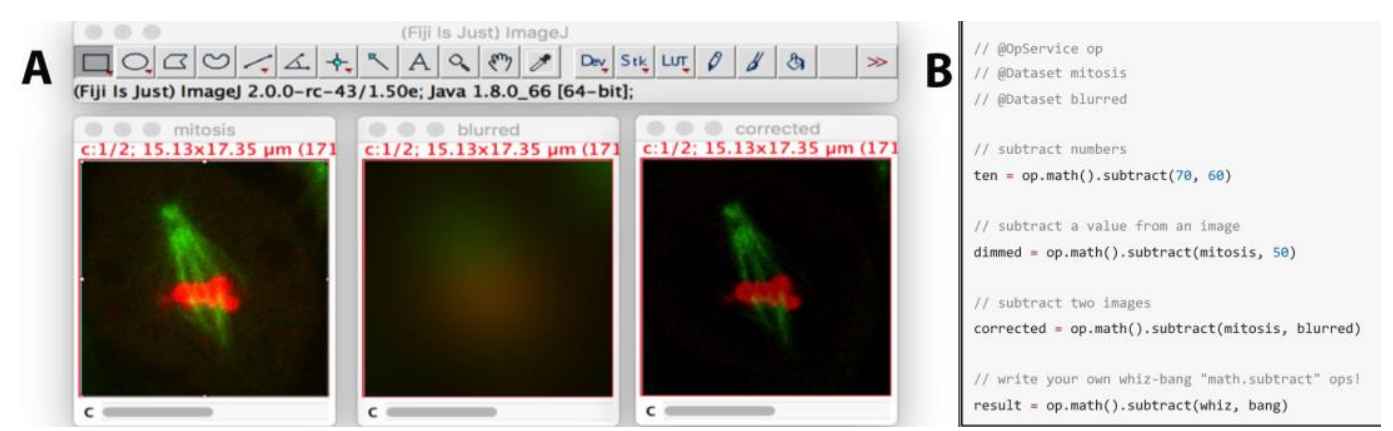

Figure 1. Screen shot of ImageJ2 in FIJI. Panel A shows the interface and a series of image processing operations using ImageJ Ops. Panel B shows the ImageJ Ops functions that created this. 